\title{
Extended Space-Time Block Coding with Transmit Antenna Selection over Correlated Fading Channels
}

\author{
Meixia Tao, Qiang Li and Hari K. Garg
}

\begin{abstract}
A new space-time block coded transmit antenna selection scheme over spatially correlated fading channels is presented. This scheme allows two or more transmit antennas to simultaneously use one radio frequency frontend. A system with four transmit antennas is considered in particular. The four antennas are selectively grouped into two subsets. Alamouti code is then applied on top of the subsets as if each was a single antenna. This scheme is shown to be more efficient than the conventional transmit antenna selection combined with Alamouti code in correlated channels. Moreover, it lowers the bitrate of the feedback channel.
\end{abstract}

\section{Index Terms}

Transmit antenna selection, space-time block code, diversity, spatial correlation

\section{INTRODUCTION}

Multiple-input multiple-output (MIMO) antenna systems provide significant enhancement on data rate and link reliability. This enhancement comes along with extra complexity, size and price in system hardware design, since each antenna port is required to connect with one radiofrequency $(\mathrm{RF})$ chain. Antenna selection techniques that can reduce the cost and attain many of the advantages of MIMO systems are therefore of great interest [1]. Of the existing selection

Meixia Tao and Hari K. Garg are with the Department of Electrical and Computer Engineering, National University of Singapore, Singapore 117576. Email: \{mxtao, eleghk\}@nus.edu.sg. Qiang Li was an undergraduate in the Department of Electrical and Computer Engineering, National University of Singapore, Singapore 117576 
techniques, transmit antenna selection (TAS) is an important category. Using TAS, one antenna or a subset of the antennas are selected for transmission. It is shown in [2]-[4] that, in a TASbased MIMO system, full diversity benefits can be retained as if all the transmit antennas were in use. Recently, numerous TAS schemes combined with space-time coding have been proposed [2], [3], [5]. In particular, Gore et al. proposed to combine TAS with Alamouti space-time block code (STBC) [6], in which two out of all transmit antennas are selected to transmit data using Alamouti STBC. We refer to this scheme as TAS/STBC. It is worthwhile to point out that all the existing antenna selection schemes assume by default that there is a one-to-one mapping between the selected antennas and the available RF chains. In practice, due to insufficient antenna spacing or surrounding scatterers, spatial correlation occurs between the path gains associated with different transmit-receive antenna pairs. Strong spatial correlation incurs substantial loss in channel capacity [7]. With transmit antenna correlation, as the degree of randomness to be exploited via antenna selection decreases, TAS schemes may not be as effective as when the transmit antennas are independent.

In this work, we introduce a new transmit antenna selection scheme tailored for spatially correlated fading channels. This scheme allows two or more transmit antennas to simultaneously use a common RF chain and hence to transmit identical signal streams. The transmitter hardware cost is almost the same as that of traditional transmit antenna selection schemes. An exemplary system with four transmit antennas is considered. The antennas are selectively grouped into two subsets, each consisting of two antennas and connecting with one RF chain. Alamouti STBC [6] is then applied on top of the two subsets as if each was a single antenna. The proposed scheme is referred to as transmit antenna selection using extended STBC or TAS/ESTBC. Our grouping criterion is to maximize the instantaneous received signal-to-noise ratio (SNR) for each transmitted symbol, and correspondingly to minimize the probability of symbol error. Simulation results show that the proposed scheme can provide up to $2 \mathrm{~dB}$ improvement over the conventional TAS/STBC scheme [2] in transmit correlated fading channels, while requiring less bandwidth for the feedback channel.

The rest of this Letter is organized as follows. In Section II we present the channel model and transmitter and receiver structures of the proposed TAS/ESTBC. Performance comparison with existing schemes is carried out in Section III. In Section IV some related work and discussions are presented. Finally, we draw conclusions in Section V. 


\section{Extended Space Time Block Coding}

\section{A. Channel Model}

We consider a narrow-band communication link with $M$ transmit antennas and $N$ receive antennas. The channel is assumed to be frequency-nonselective Rayleigh fading. It remains approximately constant during a transmission frame but can vary from one frame to another. We further consider that the antennas at both communication sides are not necessarily placed sufficiently apart from each other so that spatial correlation may occur. To focus on the effect of transmit antenna correlation on the performance of transmit antenna selection, we adopt the "one-ring" channel model in [7, Fig. 1], where the transmitter (assumed to be the base station) is elevated and unobstructed, whereas the receiver (assumed to be the mobile user) is surrounded by local scatterers. For this channel model, we let the antenna spacing at the receiver side be large enough that the fades associated with different receive antennas are independent, but those associated with different transmit antennas are correlated. Mathematically, let $\mathbf{H}$ be the $N \times M$ channel matrix, of which the $(n, m)$-th element, $h_{n, m}$, is the channel coefficient for the path from the $m$-th transmit antenna to the $n$-th receive antenna. Then, the $N$ rows of the channel matrix $\mathbf{H}$ can be modeled as independent and identically distributed (i.i.d.) complex Gaussian row vectors with covariance matrix $\mathbf{R}_{T}$. The $\left(m, m^{\prime}\right)$-th element of $\mathbf{R}_{T}$ is defined as $\mathbf{R}_{T}\left(m, m^{\prime}\right)=\mathcal{E}\left[h_{n, m} h_{n, m^{\prime}}^{*}\right]$, where notation $\mathcal{E}$ stands for expectation. Let $\mathbf{B}$ be a square root of the matrix $\mathbf{R}_{T}$ so that $\mathbf{B B}^{H}=\mathbf{R}_{T}$, with the superscript ${ }^{H}$ denoting conjugate transpose. The statistical properties of $\mathbf{H}$ are then identical to those of the product matrix $\hat{\mathbf{H}} \mathbf{B}^{H}$. Here, $\hat{\mathbf{H}}$ is an $N \times M$ matrix with i.i.d. complex Gaussian entries of zero mean and unit variance. We shall use this statistical equivalence to generate each channel realization in our numerical analysis in Section III. Note that this method has been widely used in [2], [7].

\section{B. Extended STBC with Transmit Antenna Selection}

The transmitter architecture of the proposed scheme for $M=4$ transmit antennas using two $\mathrm{RF}$ frontends is depicted in Fig. 1. The STBC signals generated in the baseband processor are

up-converted to a radio channel by using two RF chains. A typical RF chain consists of a digital-to-analog converter, a frequency up-converter and a power amplifier. The passband signal stream from each chain is then split into two sub-streams with the aid of a power divider. The 
mapping between the four transmit antennas and the four RF signal sub-streams is implemented in the switch unit. The receiver informs the transmitter of the switch setting according to the latest channel state information through a feedback channel with limited capacity. The baseband equivalence of the resulting passband signals transmitted on the four antennas within two symbol intervals can be represented by a $4 \times 2$ codeword matrix:

$$
\mathbf{X}=\sqrt{\frac{E_{s}}{4}}\left[\begin{array}{cc}
s_{1} & -s_{2}^{*} \\
s_{1} & -s_{2}^{*} \\
s_{2} & s_{1}^{*} \\
s_{2} & s_{1}^{*}
\end{array}\right] .
$$

In (1), $s_{1}$ and $s_{2}$ are two independent complex-valued information symbols with unit average energy, and $E_{s} / 4$ is the radiation power on each antenna. The factor $1 / 4$ is to assure that the system total radiation power is independent of the number of transmit antennas and equal to $E_{s}$. The matrix in (1) is called an extended space-time block code (ESBTC).

Let $\tilde{\mathbf{H}}=\left[\tilde{\mathbf{h}}_{1}, \ldots \tilde{\mathbf{h}}_{4}\right]$ denote the effective channel matrix seen by the ESTBC codeword (1), obtained by swapping the columns of the original channel matrix $\mathbf{H}=\left[\mathbf{h}_{1}, \ldots, \mathbf{h}_{4}\right]$ according to the control bits from the feedback channel. The baseband received signals on the $N$ receive antennas at the two time slots, denoted by $N \times 1$ vectors $\mathbf{y}_{1}$ and $\mathbf{y}_{2}$ respectively, can thus be written as:

$$
\begin{aligned}
\mathbf{y}_{1} & =\sqrt{\frac{E_{s}}{4}}\left(\tilde{\mathbf{h}}_{1}+\tilde{\mathbf{h}}_{2}\right) s_{1}+\sqrt{\frac{E_{s}}{4}}\left(\tilde{\mathbf{h}}_{3}+\tilde{\mathbf{h}}_{4}\right) s_{2}+\mathbf{w}_{1} \\
\mathbf{y}_{2} & =\sqrt{\frac{E_{s}}{4}}\left(\tilde{\mathbf{h}}_{1}+\tilde{\mathbf{h}}_{2}\right)\left(-s_{2}^{*}\right)+\sqrt{\frac{E_{s}}{4}}\left(\tilde{\mathbf{h}}_{3}+\tilde{\mathbf{h}}_{4}\right) s_{1}^{*}+\mathbf{w}_{2} .
\end{aligned}
$$

Here, $\mathbf{w}_{i}$, for $i=1,2$, is the complex additive white Gaussian noise vector with each entry being independent and having mean zero and variance $N_{0} / 2$ per dimension.

Similar to the original Alamouti STBC, the maximum likelihood decision variables for symbols $s_{1}$ and $s_{2}$ are computed, respectively, as

$$
\hat{s}_{1}=\left(\tilde{\mathbf{h}}_{1}+\tilde{\mathbf{h}}_{2}\right)^{H} \mathbf{y}_{1}+\mathbf{y}_{2}^{H}\left(\tilde{\mathbf{h}}_{3}+\tilde{\mathbf{h}}_{4}\right)
$$

and

$$
\hat{s}_{2}=\left(\tilde{\mathbf{h}}_{3}+\tilde{\mathbf{h}}_{4}\right)^{H} \mathbf{y}_{1}-\mathbf{y}_{2}^{H}\left(\tilde{\mathbf{h}}_{1}+\tilde{\mathbf{h}}_{2}\right) .
$$


Thus, the instantaneous received SNR for each symbol is

$$
\begin{aligned}
\gamma_{22} & =\frac{\gamma_{0}}{4}\left[\left\|\tilde{\mathbf{h}}_{1}+\tilde{\mathbf{h}}_{2}\right\|^{2}+\left\|\tilde{\mathbf{h}}_{3}+\tilde{\mathbf{h}}_{4}\right\|^{2}\right] \\
& =\frac{\gamma_{0}}{4}\left[\sum_{m=1}^{4}\left\|\mathbf{h}_{m}\right\|^{2}+2 \operatorname{Re}\left\{\tilde{\mathbf{h}}_{1}^{H} \tilde{\mathbf{h}}_{2}+\tilde{\mathbf{h}}_{3}^{H} \tilde{\mathbf{h}}_{4}\right\}\right],
\end{aligned}
$$

in which $\gamma_{0} \triangleq E_{s} / N_{0}$ is the system total transmit $\mathrm{SNR},\|\cdot\|^{2}$ and $\operatorname{Re}\{\cdot\}$ represent the squared Euclidean norm and the real part, respectively. The subscript in $\gamma_{22}$ stands for the 2-2 grouping as in Fig. 1.

Clearly, to maximize the received SNR $\gamma_{22}$ in (2), and correspondingly to minimize the instantaneous probability of symbol error for a given channel realization $\mathbf{H}$, one should choose a swapping $\left\{\tilde{\mathbf{h}}_{1}, \tilde{\mathbf{h}}_{2}, \tilde{\mathbf{h}}_{3}, \tilde{\mathbf{h}}_{4}\right\}$ that produces the largest value of $\operatorname{Re}\left\{\tilde{\mathbf{h}}_{1}^{H} \tilde{\mathbf{h}}_{2}+\tilde{\mathbf{h}}_{3}^{H} \tilde{\mathbf{h}}_{4}\right\}$. Since the antenna order in the subset $\left\{\tilde{\mathbf{h}}_{1}, \tilde{\mathbf{h}}_{2}\right\}$ and the order in $\left\{\tilde{\mathbf{h}}_{3}, \tilde{\mathbf{h}}_{4}\right\}$ are insignificant, due to the $\mathrm{RF}$ sharing architecture, the criterion is equivalent to choosing a transmit antenna grouping $\left\{\left(\tilde{\mathbf{h}}_{1}, \tilde{\mathbf{h}}_{2}\right),\left(\tilde{\mathbf{h}}_{3}, \tilde{\mathbf{h}}_{4}\right)\right\}$ with maximum $\operatorname{Re}\left\{\tilde{\mathbf{h}}_{1}^{H} \tilde{\mathbf{h}}_{2}\right\}+\operatorname{Re}\left\{\tilde{\mathbf{h}}_{3}^{H} \tilde{\mathbf{h}}_{4}\right\}$. Note that $\operatorname{Re}\left\{\mathbf{h}_{m}^{H} \mathbf{h}_{m^{\prime}}\right\}$ indicates the similarity between the instantaneous channel gain vectors on $m$-th and $m^{\prime}$-th transmit antennas. Intuitively, higher statistical correlation among the transmit antennas would yield a higher chance of having high instantaneous similarity. As a result, more antenna array gain can be expected.

\section{PERFORMANCE COMPARISON}

In this section we illustrate the performance of the proposed TAS/ESTBC scheme by comparing it with the conventional TAS/STBC scheme in average received SNR gain, symbol error rate (SER) performance, and the number of feedback bits required.

\section{A. Average SNR Gain}

Recall that the baseband transmitted codeword in TAS/STBC using the same total radiation power $E_{s}$ as in the proposed scheme can be described by the matrix

$$
\mathbf{A}=\sqrt{\frac{E_{s}}{2}}\left[\begin{array}{cc}
s_{1} & -s_{2}^{*} \\
s_{2} & s_{1}^{*}
\end{array}\right] .
$$

The instantaneous received SNR of each symbol after detection is given by [2]

$$
\gamma_{2 / 4}=\frac{\gamma_{0}}{2}\left[\left\|\mathbf{h}_{\max 1}\right\|^{2}+\left\|\mathbf{h}_{\max 2}\right\|^{2}\right]
$$


where $\mathbf{h}_{\max 1}$ and $\mathbf{h}_{\max 2}$ are the two columns of the channel matrix $\mathbf{H}$ with the largest Euclidean norms, and the subscript in $\gamma_{2 / 4}$ indicates a 2-out-of-4 transmit antenna selection. The quantity $\gamma_{0}$ in (4) is defined in the same way as in (2).

We first compare the average received SNRs of TAS/ESTBC $\bar{\gamma}_{22}$ and TAS/STBC $\bar{\gamma}_{2 / 4}$ in two extreme cases. In the extreme case where $\left\{\mathbf{h}_{m}\right\}$ are fully correlated and $\mathbf{h}_{m}=\mathbf{h}, \forall m$, one can have $\gamma_{22}=2\|\mathbf{h}\|^{2} \gamma_{0}$ and $\gamma_{2 / 4}=\|\mathbf{h}\|^{2} \gamma_{0}$. Hence, $\bar{\gamma}_{22}$ is 3 -dB higher than $\bar{\gamma}_{2 / 4}$. This is explained as follows. The strategy of transmitting identical signal streams on the two antennas within each subset in our proposed TAS/ESTBC scheme can be regarded as a special case of beamforming, where the beamforming coefficients are all equal to 1 . This strategy is optimal when the channel gains associated with the two antennas are the same. Therefore, a beamforming gain of $3 \mathrm{~dB}$ in this extreme case is achieved. In the other extreme case where $\left\{\mathbf{h}_{m}\right\}$ are independent (no transmit correlation), since the maximum value of $\operatorname{Re}\left\{\tilde{\mathbf{h}}_{1}^{H} \tilde{\mathbf{h}}_{2}+\tilde{\mathbf{h}}_{3}^{H} \tilde{\mathbf{h}}_{4}\right\}$ in $\gamma_{22}$ over all the grouping methods may still be negative, full diversity is not guaranteed in TAS/ESTBC. Therefore, the proposed TAS/ESTBC scheme is inferior to the TAS/STBC scheme when the channels are independent.

In the general case where the transmit antennas are arbitrarily correlated, it is difficult to evaluate the probability density functions of $\gamma_{22}$ and $\gamma_{2 / 4}$. Thus, we resort to numerical methods for computing both $\bar{\gamma}_{22}$ and $\bar{\gamma}_{2 / 4}$ via simulation. For simplicity, we assume an evenly correlated channel where the correlation coefficient between any pair of transmit antennas is the same and denoted as $\rho$. Let $g_{22}=10 \log _{10}\left[\bar{\gamma}_{22} /\left(\gamma_{0} N\right)\right]$ and $g_{2 / 4}=10 \log _{10}\left[\bar{\gamma}_{2 / 4} /\left(\gamma_{0} N\right)\right]$ denote the gain in average received SNR of the proposed TAS/ESTBC scheme and the conventional TAS/STBC scheme, respectively. Fig. 2 shows the gains at different values of $\rho$ and $N$. Each gain value is obtained by averaging over $10^{5}$ independent channel realizations. It is observed that at low transmit antenna correlation, the gain of TAS/ESTBC is lower than that of TAS/STBC. As $\rho$ increases, the gain of TAS/ESTBC increases, whereas the gain of TAS/STBC decreases. In the extreme case with $\rho=1$ (fully correlated), $g_{22}$ becomes $3 \mathrm{~dB}$ larger than $g_{2 / 4}$. This implies that the conventional TAS/STBC is more suitable when the transmit antennas are independent or have low correlation, but our scheme is more beneficial when the antenna correlation is medium or high. From Fig. 2 one can also observe that, at large $\rho(0.4<\rho<1)$, as the number of receive antenna increases the gap between $g_{22}$ and $g_{2 / 4}$ also increases. Thus, it can be expected that the error performance gain of TAS/ESTBC over TAS/STBC is higher with more receive antennas, but is always upper bounded by $3 \mathrm{~dB}$. 


\section{B. SER Performance}

The above analysis based on the average received SNR serves as a good performance indicator, but is not sufficient for the comparison of average probability of error. In this subsection we provide simulation results to compare the symbol error rate between the proposed TAS/ESTBC scheme and the conventional TAS/STBC scheme.

The communication link follows the "one-ring" model and is equipped with a linear array consisting of $M=4$ equally spaced antennas at the transmitter and with $N=1$ or 2 independent antennas at the receiver. It is assumed that the angle of departure is perpendicular to the transmit antenna array and that the angle spread (denoted by $\Delta$ ) is small. Thus, by using the results in [7], the spatial correlation between the $m$-th and $m^{\prime}$-th transmit antennas can be approximated by $\mathbf{R}_{\mathrm{T}}\left(m, m^{\prime}\right) \approx J_{0}\left(2 \pi \Delta\left|m-m^{\prime}\right| d / \lambda\right)$, where $\lambda$ is the carrier wavelength, $d$ is the spacing between two adjacent antenna elements and $J_{0}(\cdot)$ is the zeroth-order Bessel function of the first kind. In our simulation, we fix the angle spread $\Delta$ to 0.6 degree and let the antenna spacing $d$ vary.

Figs. 3 and 4 illustrate the symbol error rate results with QPSK modulation at various values of $d$ using 1 receive antenna and 2 receive antennas, respectively. The total transmit SNR per bit is defined as $\gamma_{0} / b$, where $b$ is the transmission rate in bits/s/Hz. For comparison, we also provide the results for the open-loop rate-3/4 STBC with 4 transmit antennas [8, Eqn.(41)]. Note that the transmission rate of the open-loop STBC is only $1.5 \mathrm{bits} / \mathrm{s} / \mathrm{Hz}$ due to the non-full-rate code design, whereas the rate of the two transmit antenna selection schemes is 2 bits/s/Hz. Moreover, the open-loop scheme requires four RF chains at the transmitter, but only two RF chains are needed in the selection schemes. From Figs. 3 and 4, we can have several observations. Firstly, in the independent channels, the performance of the proposed TAS/ESTBC scheme is about 0.8 $\mathrm{dB}$ worse than that of TAS/STBC for both one and two receive antennas. This is higher than the corresponding $0.5 \mathrm{~dB}$ and $0.4 \mathrm{~dB}$ differences in the average received SNR gains observed from Fig. 2 for $\rho=0$. Secondly, as the transmit antenna correlation increases, by decreasing the antenna spacing $d$, the proposed TAS/ESTBC scheme becomes superior to TAS/STBC. Moreover, the performance improvement using two receive antennas is larger than the gain using one receive antenna. In particular, an improvement of about $2 \mathrm{~dB}$ at all SNR regions can be observed when $d=2 \lambda$ for two receive antennas. On the other hand, an improvement of $2.6 \mathrm{~dB}$ in average 
received SNR is seen from Fig. 2 for $\rho=0.96$ (the smallest antenna correlation coefficient for $d=2 \lambda$ ). We can see that the performance trend of the symbol error rate results matches very well with the comparison on average received SNRs, but the gains predicted in Fig. 2 are slightly optimistic. In addition, it is also observed that both the transmit antenna selection schemes with limited feedback outperform the open-loop STBC. But the performance gain of the conventional TAS/STBC over the open-loop scheme decreases as the antenna correlation increases. For instance, when two receive antennas are used, the gain at a target SER of $10^{-4}$ is around $1.5 \mathrm{~dB}$ for independent channels, but it reduces to $0.7 \mathrm{~dB}$ or less in correlated channels with $d=2 \lambda$. In contrast, the gain of the proposed TAS/ESTBC over the open-loop scheme increases as the antenna correlation increases. These results further confirm the benefits of using the proposed scheme in spatially correlated fading channels.

\section{Feedback Bits}

The number of information bits needed on the feedback path is also different for the two transmit antenna selection schemes. In the proposed TAS/ESTBC scheme, the legitimate antenna subset grouping methods include $\left\{\left(\mathbf{h}_{1}, \mathbf{h}_{2}\right),\left(\mathbf{h}_{3}, \mathbf{h}_{4}\right)\right\},\left\{\left(\mathbf{h}_{1}, \mathbf{h}_{3}\right),\left(\mathbf{h}_{2}, \mathbf{h}_{4}\right)\right\}$, and $\left\{\left(\mathbf{h}_{1}, \mathbf{h}_{4}\right),\left(\mathbf{h}_{2}, \mathbf{h}_{3}\right)\right\}$. Thus, the feedback information required is $\log _{2} 3=1.585$ bits. In the conventional TAS/STBC scheme, the total number of ways of selecting two out of the four transmit antennas is $C_{4}^{2}=6$, which requires one more bit in the feedback path than our new scheme does.

\section{RELATED WORK AND Discussions}

Transmit antenna selection is of practical as well as fundamental importance because of its low hardware cost and high diversity gain. To implement TAS, a feedback channel must be present to send the selection information according to the latest channel estimates. In the ideal case where the feedback channel is error-free and delay-free and the antennas are all independent, the optimal selection scheme that maximizes the instantaneous received SNR is to select one and only one transmit antenna [9]. However, in practice, it is not feasible that the best antenna can always be selected due to feedback delay, feedback errors and channel estimation errors. The results in [9] and [10] show that the antenna subset selection is more robust to imperfect selection than the single antenna selection. Therefore, the proposed scheme in this work that 
employs antenna subset grouping can also perform robustly in a more realistic environment where the feedback channel is imperfect.

The most relevant work to our scheme is [11], in which a similar antenna grouping method via RF sharing is introduced. However, as independent fading channels are assumed in [11], the RF sharing scheme is ineffective if used alone and has to be combined together with TAS/STBC as a hybrid transmit antenna/code selection scheme. The drawbacks of using such a hybrid scheme are two-fold: more feedback bits (more selection combinations) and more complicated hardware design (both RF sharing and conventional TAS/STBC should be implemented).

To enhance the system performance by employing a feedback channel with limited capacity, one can design not only TAS schemes using one or a subset of antennas but also beamforming schemes which use all the antennas, such as [12]-[14]. In particular, STBC-based group-coherent codes are proposed in [14] to achieve both diversity gain and partial beamforming gain via limited channel feedback. It is noted that the passband transmitted signal structure of this scheme is similar to ours. The work in [14] is, however, fundamentally different from the proposed scheme. Unlike the proposed scheme, the baseband signals to be transmitted within each antenna group in [14] are not identical due to beamforming phase components and, hence, there is no reduction in the number of RF frontends. Furthermore, the mapping from signals to antennas in [14] is fixed by design, whereas the signal-to-antenna mapping in the proposed scheme is adaptive relative to the time-varying channel conditions.

\section{CONCLUSION}

Extended space-time block coding with four transmit antennas was proposed over spatially correlated MIMO fading channels. By grouping the transmit antennas according to their similarities in instantaneous channel coefficients into two subsets and treating each subset as a single antenna, both hardware complexity reduction and antenna array gain can be achieved. Compared with the conventional space-time block coded transmit antenna selection, this new scheme not only achieves better error performance but also requires less capacity for the feedback channel. For systems with more than four transmit antennas our scheme can be easily applied by grouping more antennas in each subset, as long as high transmit antenna correlation is present. 


\section{REFERENCES}

[1] S. Sanayei and A. Nosratinia, "Antenna selection in MIMO systems," IEEE Comm. Magazine, vol. 42, no. 10, pp. 68-72, Oct 2004.

[2] D. A. Gore and A. J. Paulraj, "MIMO antenna subset selection with space-time coding," IEEE Trans. on Signal Processing, vol. 50, no. 10, pp. 2580-2588, Oct 2002.

[3] Z. Chen, J. Yuan, B. Vucetic, and Z. Zhou, "Performance of Alamouti scheme with transmit antenna selection," Electronics Letters, pp. 1666-1667, 2003.

[4] S. Thoen, L. Perre, B. Gyselinckx, and M. Engels, "Performance analysis of combined transmit-SC/receive-MRC," IEEE Trans. on Comm., vol. 49, no. 1, pp. 5-8, Jan 2001.

[5] Z. Chen, B. Vucetic, and J. Yuan, "Space time trellis codes with transmit antenna selection," Electronics letters, pp. 854-855, 2003.

[6] S. M. Alamouti, "A simple transmit diversity technique for wireless communication," IEEE Journal on Selected Areas in Comm., vol. 16, pp. 1451-1458, 1998.

[7] D. S. Shiu, G. J. Foschini, M. J. Gans, and J. M. Kahn, "Fading correlation and its effect on the capacity of multielement antenna systems," IEEE Trans. on Comm., vol. 48, no. 3, pp. 502-513, March 2000.

[8] G. Ganesan and P. Stoica, "Space-time block codes: a maximum SNR approach," IEEE Trans. on Info. Theory, vol. 47, no. 4, pp. 1650-1656, May 2001.

[9] J. Tang, X. Zhang, and Q. Du, "Alamouti scheme with joint antenna selection and power allocation over Rayleigh fading channels in wireless networks," in Proc. IEEE Globecom'05, 2005.

[10] Z. Chen, B. Vucetic, and J. Yuan, "Asymptotic performance of space-time block codes with imperfect transmit antenna selection," IEE Electronic Letters, vol. 41, no. 9, April 2005.

[11] R. Machado and B. F. Uchoa-Filho, "Space-time block coding with hybrid transmit antenna/code slection," in Proc. IEEE International Conference on Comm. (ICC), Paris, France, June 2004.

[12] K. K. Mukkavilli, A. Sabharwal, E. Erkip, and B. Aazhang, "On beamforming with finite rate feedback in multiple antenna systems," IEEE Trans. on Info. Theory, vol. 49, pp. 2562-2579, Oct. 2003.

[13] R. W. Heath and A. J. Paulraj, "A simple scheme for transmit diversity using partial channel feedback," in Proc. 32nd Asilomar Conf. Signals, Systems, and Computers, 1998.

[14] J. Akhtar and D. Gesbert, "Extending orthogonal block codes with partial feedback," IEEE Trans. on Wirleess Comm., vol. 3, no. 6, pp. 1959-1962, Nov. 2004. 


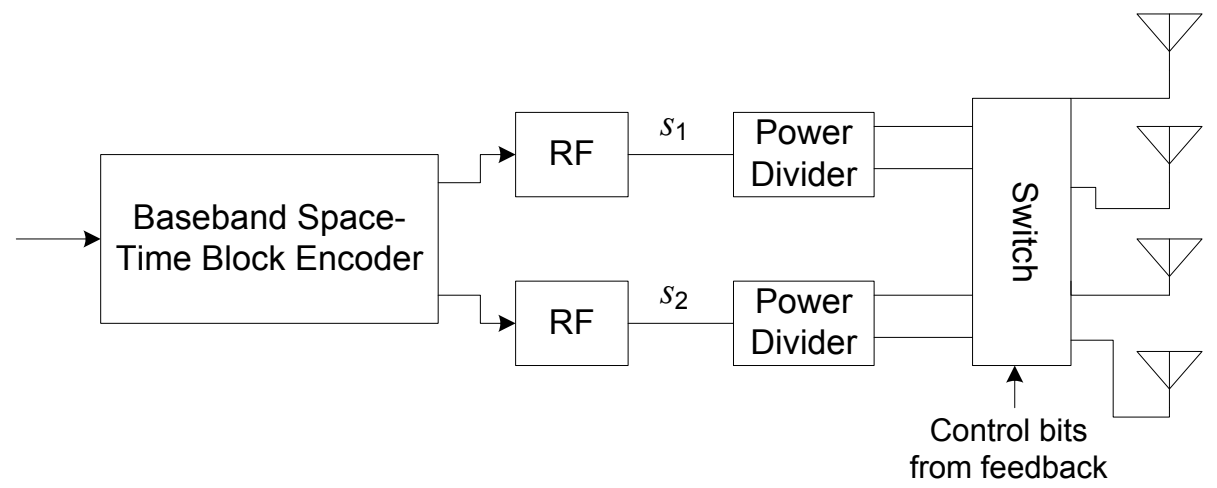

Fig. 1. Simplified transmit diagram of extended space-time block codes using two RF chains and four antennas.

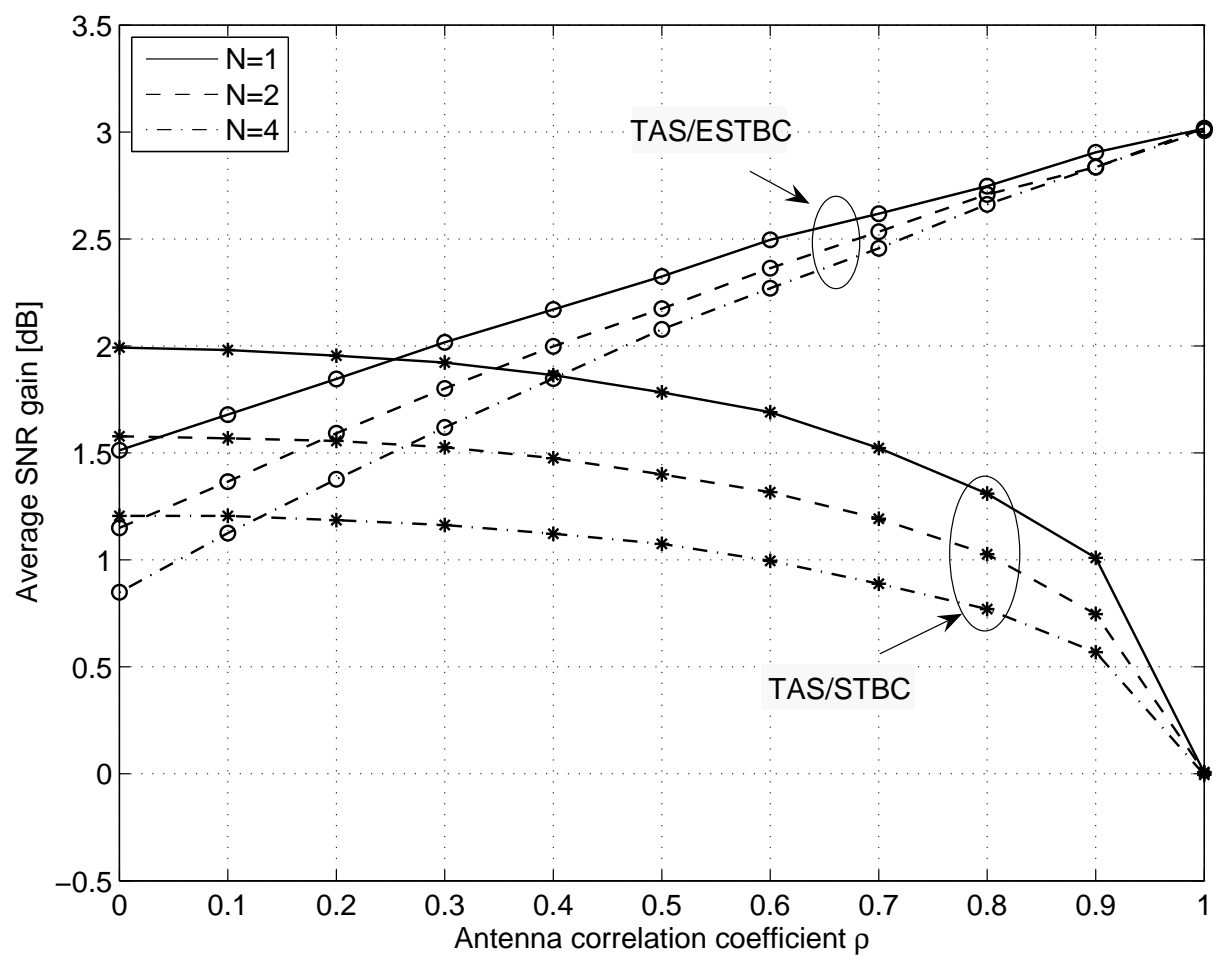

Fig. 2. Average received SNR gain comparison. 


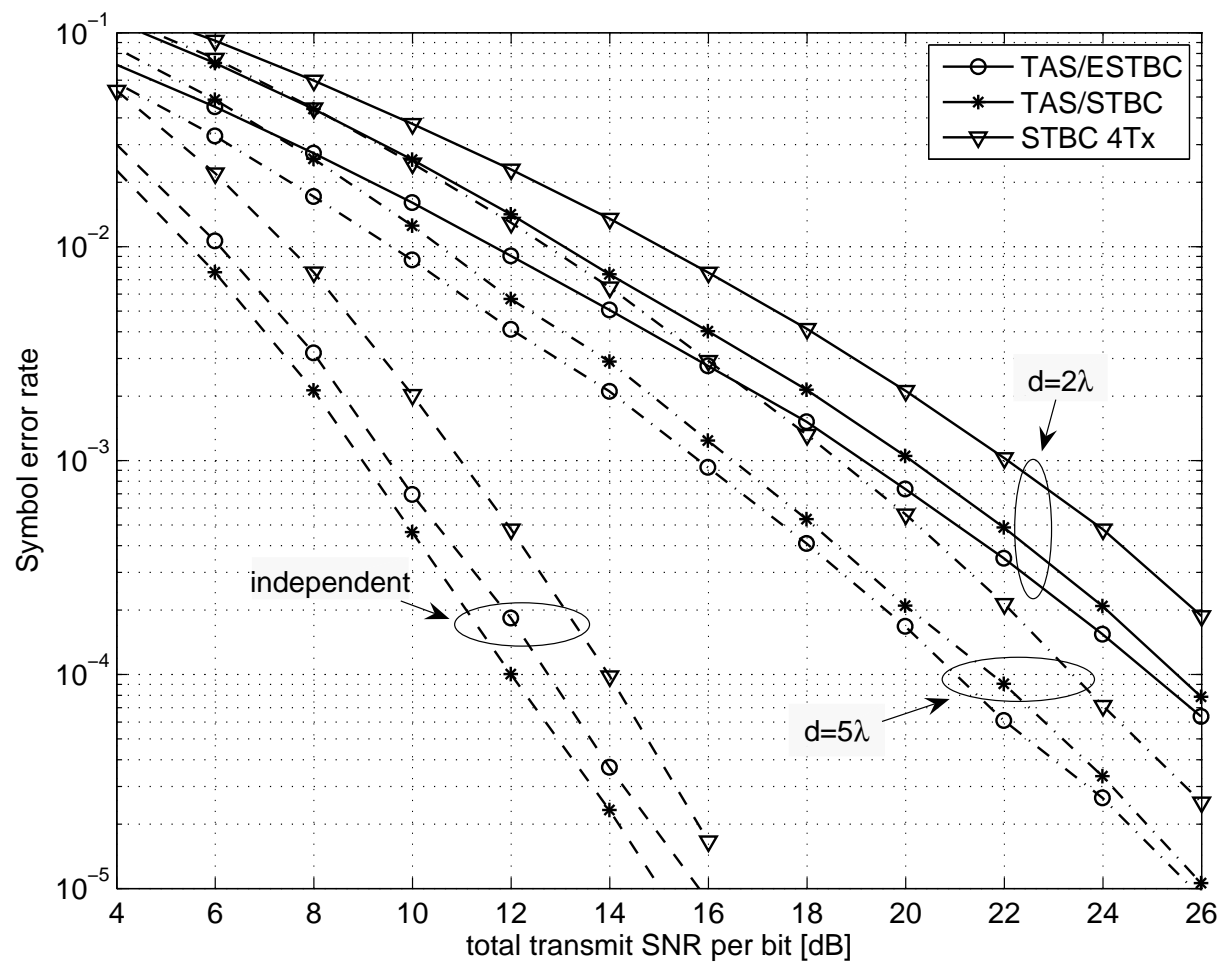

Fig. 3. Symbol error rate comparison using one receive antenna and QPSK modulation.

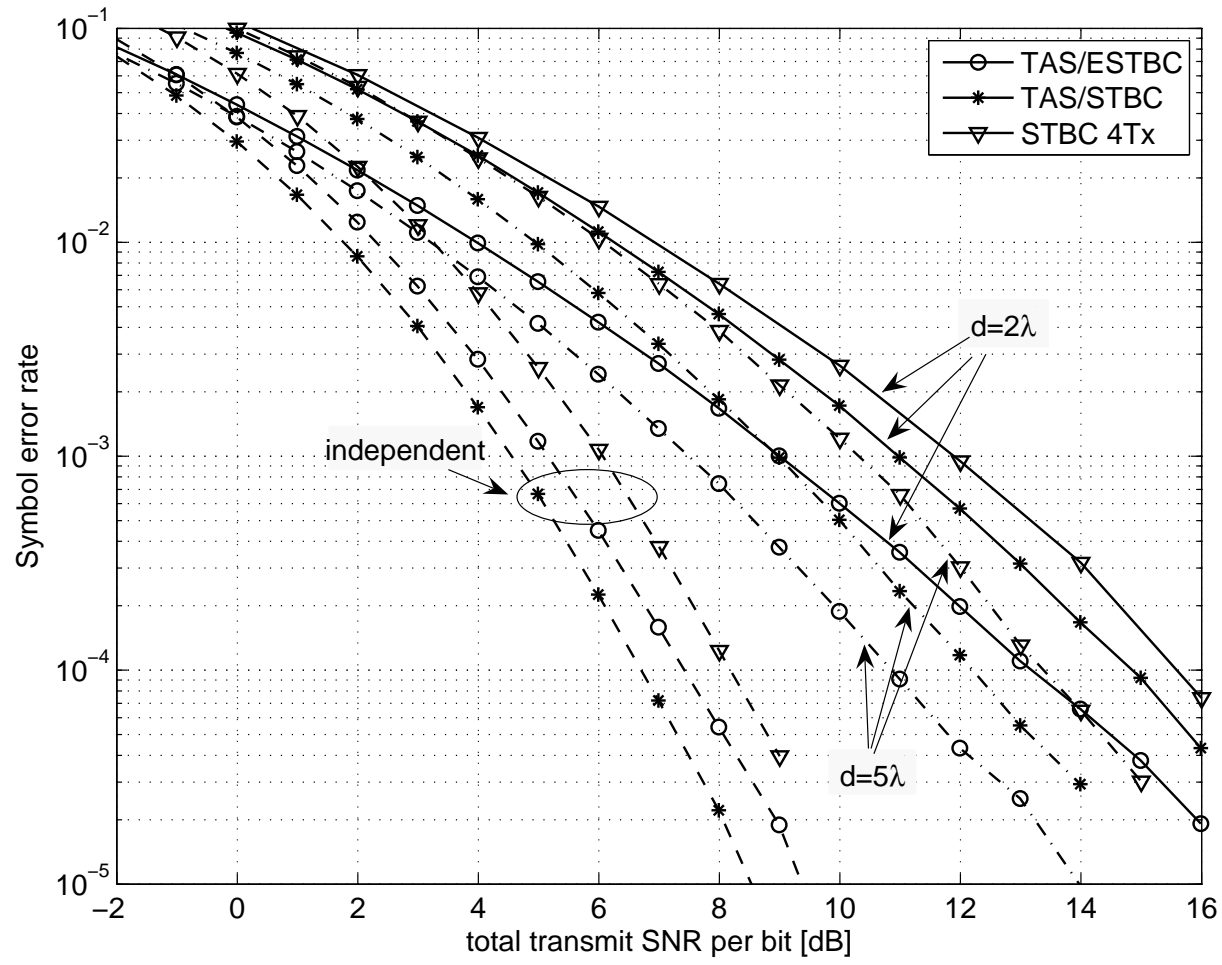

Fig. 4. Symbol error rate comparison using two receive antennas and QPSK modulation. 